\title{
Thin-film Sb2Se3 photovoltaics with oriented one-dimensional ribbons and benign grain boundaries
}

\author{
Ying Zhou, Liang Wang, Shiyou Chen, Sikai Qin, Xinsheng Liu, \\ Jie Chen, Ding-Jiang Xue, Miao Luo, Yuanzhi Cao, Yibing Cheng, \\ Edward H. Sargent \& Jiang Tang
}

Version Post-Print/Accepted Manuscript

Citation Zhou, Y., Wang, L., Chen, S., Qin, S., Liu, X., Chen, J., Xue, D.-J., Luo, (published version) M., Cao, Y., Cheng, Y., Sargent, E. H., and Tang, J. (2015). Thin-film Sb2Se3 photovoltaics with oriented one-dimensional ribbons and benign grain boundaries. Nature Photonics, 9(6), 409-415.

doi:10.1038/nphoton.2015.78

Publisher's Statement The final published version of this article is available at Nature

Photonics via http://dx.doi.org/10.1038/nphoton.2015.78.

\section{How to cite TSpace items}

Always cite the published version, so the author(s) will receive recognition through services that track citation counts, e.g. Scopus. If you need to cite the page number of the TSpace version (original manuscript or accepted manuscript) because you cannot access the published version, then cite the TSpace version in addition to the published version using the permanent URI (handle) found on the record page. 


\title{
Thin-film $\mathrm{Sb}_{2} \mathrm{Se}_{3}$ photovoltaics with oriented $1 \mathrm{D}$ ribbons and benign grain boundaries
}

\author{
Ying Zhou ${ }^{1,2}$, Liang Wang ${ }^{1,2}$, Shiyou Chen ${ }^{3}$, Sikai Qin ${ }^{1,2}$, Xinsheng Liu ${ }^{1,2}$, Jie Chen ${ }^{1,2}$, \\ Ding-Jiang Xue ${ }^{1,2}$, Miao Luo ${ }^{1,2}$, Yuanzhi Cao ${ }^{1}$, Yibing Cheng ${ }^{1}$, Edward H. Sargent ${ }^{4}$, Jiang Tang ${ }^{1,2 *}$ \\ ${ }^{1}$ Wuhan National Laboratory for Optoelectronics (WNLO), and ${ }^{2}$ School of Optical and Electronic \\ Information, Huazhong University of Science and Technology (HUST), Wuhan, 430074, China \\ 3. Key Laboratory for Polar Materials and Devices (MOE), East China Normal University, Shanghai \\ 200241, China \\ ${ }^{4}$ Department of Electrical and Computer Engineering, University of Toronto, Toronto, Ontario, M5S \\ 3G4, Canada
}

Correspondence and requests for materials should be addressed to J. T. (E-mail: jtang@mail.hust.edu.cn)

Solar cells based on inorganic absorbers such as $\mathrm{Si}$, GaAs, CdTe and $\mathrm{Cu}(\mathrm{In}, \mathrm{Ga}) \mathrm{Se} 2$ permit high device efficiency and stability. Because of the crystals' three dimensional (3D) structure, dangling bonds inevitably exist at the grain boundaries (GBs), significantly degrading device performance via recombination losses. Thus, growth of single-crystalline materials or the passivation of defects at GBs are required to address this problem, which introduces added processing complexity and cost. Here we report that antimony selenide (Sb2Se3) - a simple, non-toxic and low-cost material with optimal solar bandgap of $\sim 1.1 \mathrm{eV}$ - exhibits intrinsically benign GBs because of its one dimensional (1D) crystal structure. Using a simple and fast $(\sim 1 \mu \mathrm{m} / \mathrm{min})$ rapid thermal evaporation process, we orient crystal growth perpendicular to the substrate, and produce Sb2Se3 thin-film solar cells with $5.6 \%$ certified device efficiency. Our results suggest that the family of $1 \mathrm{D}$ crystals including Sb2S3, SbSeI and Bi2S3 show promise in photovoltaic applications.

The urgent need for high-efficiency, low-cost solar cells drives sustained research on new absorber materials for thin film photovoltaics. Copper zinc tin sulfide (CZTS) and organic-inorganic metal halide perovskites $\left(\mathrm{CH}_{3} \mathrm{NH}_{3} \mathrm{PbI}_{3}\right)$ are promising absorber materials that have achieved impressive certified device efficiencies ${ }^{1,2}$. However, the complexity of defect physics associated with $\mathrm{CZTS}^{3,4}$ appears to limit further efficiency improvements in this system, and concerns over stability and reliance on lead in $\mathrm{CH}_{3} \mathrm{NH}_{3} \mathrm{PbI}_{3}{ }^{5}$ creates opportunities for new, stable, $\mathrm{Pb}$-free materials.

These and other widely-explored photovoltaic absorber materials - such as $\mathrm{Si}$, GaAs, CdTe, InP, $\mathrm{Cu}(\mathrm{In}, \mathrm{Ga}) \mathrm{Se}_{2}$ (CIGS) - exhibit a three dimensional (3D) crystal structure ${ }^{6-8}$, i.e., they are bound by covalent and/or ionic bonds in all three spatial dimensions. The 3D crystal structure guarantees isotropic carrier transport and permits relaxed orientation control. However, at discontinuities such as grain boundaries (GBs), dangling bonds typically act as recombination centers, and require further steps to be remedied ${ }^{9-11}$. In CdTe for example (Fig. 1a), recombination loss through dangling bonds at GBs lowers open-circuit voltage even when the best available passivation methods are employed ${ }^{12}$.

We hypothesized that materials possessing one dimensional (1D) crystal structure (Fig. 1b), such as 
ribboned compounds $\mathrm{Sb}_{2} \mathrm{Se}_{3}$ and $\mathrm{Bi}_{2} \mathrm{~S}_{3}$, could, if suitably oriented, offer compelling performance. In $\mathrm{Sb}_{2} \mathrm{Se}_{3}$, for example, $\left(\mathrm{Sb}_{4} \mathrm{Se}_{6}\right)_{\mathrm{n}}$ ribbons stack along the [001] direction through strong covalent $\mathrm{Sb}-\mathrm{Se}$ bonds while in the [100] and [010] direction, the $\left(\mathrm{Sb}_{4} \mathrm{Se}_{6}\right)_{\mathrm{n}}$ ribbons are held together by van der Waals forces. These materials have been rarely explored for photovoltaics because of concerns about poor carrier transport between ribbons, and worries about the mechanical stability of films.

We posited that a new materials processing strategy designed to orient ribbons vertically on the substrate could permit photogenerated carriers to travel efficiently along the covalently-bonded 1D axis. In the orthogonal direction, the parallel-stacked ribbons would provide substantially no dangling bonds, even at GBs, thereby minimizing recombination losses - an important advantage in photovoltaic application because recombination losses at GBs is one of the major limiting factors for high efficiency thin film solar cells.

We began with first-principles simulations (VASP code) to study the structural relaxation and electronic structure of $\mathrm{Sb}_{2} \mathrm{Se}_{3}$ surfaces. The available terminations of (100) and (010) surfaces in $\mathrm{Sb}_{2} \mathrm{Se}_{3}$ require no breaking of Sb-Se bonds (Fig. 1c) and thus produce no dangling bonds. Other surfaces parallel to the [001] direction also have no dangling bonds, e.g., the (110) and (120) surfaces. Their surface energy is therefore lower than those with dangling bonds, such as the (001), (211) and (221) surfaces. Calculation reveals that, as expected, the surface energies for (100), (010), (110), (120), (001), (211) and (221) surfaces, respectively, are $0.44,0.25,0.33,0.32,0.46,0.56,0.53 \mathrm{~J} / \mathrm{m}^{2}$. This indicates that the most abundant surfaces in $\mathrm{Sb}_{2} \mathrm{Se}_{3}$ samples will be the (010), (110) and (120) surfaces, i.e. those having the lowest formation energies and no breakage of covalent bonds. Density functional theory confirms quantitatively that no extra states are introduced inside the bandgap by these terminations. A clean bandgap is seen in the calculated density of states (DOS) of the four $\mathrm{Sb}_{2} \mathrm{Se}_{3}$ surfaces (Fig. 1d) and the gap is comparable to that of the bulk $\mathrm{Sb}_{2} \mathrm{Se}_{3}$. Furthermore, there is no appreciable change in the calculated DOS over a wide energy range $(-10$ to $3 \mathrm{eV})$, indicating that there is no significant reconstruction on these surfaces. In sum, the ribbons represent the fundamental building block of the $\mathrm{Sb}_{2} \mathrm{Se}_{3}$ structure, and surface reconstruction is negligible as long as this basic repeat unit is not broken.

Overall, this computational study confirms that, as long as the $\mathrm{Sb}_{2} \mathrm{Se}_{3}$ ribbons are suitably oriented, the GBs will be terminated by the intrinsically benign surfaces (e.g. (100), (010), (110) and (120) planes) and minimize recombination loss. This is in striking contrast to most known photovoltaic absorbers, in which the breakage of covalent bonds introduces defect states and recombination centers at $\mathrm{GBs}^{13-15}$.

A number of additional properties make $\mathrm{Sb}_{2} \mathrm{Se}_{3}$ particularly worthy of experimental investigation for high efficiency, low cost thin film solar cells ${ }^{16}$. Like CdTe, but in contrast with CIGS and CZTS, $\mathrm{Sb}_{2} \mathrm{Se}_{3}$ is a simple binary compound with fixed phase and stoichiometry. It has a very strong absorption coefficient ( $>10^{5} \mathrm{~cm}^{-1}$ at short wavelengths) and its bandgap is $\sim 1.1 \mathrm{eV}^{17,18}$, optimal for single-junction solar cells. The constituents of $\mathrm{Sb}_{2} \mathrm{Se}_{3}$ are non-toxic and low in cost ( $\mathrm{Sb}$ has similar cost to $\mathrm{Cu}$ ), and, as we proceed to show herein, $\mathrm{Sb}_{2} \mathrm{Se}_{3}$ films are produced using minimal energy, enabling in principle a low energy-payback time for a solar cell ${ }^{19}$. All of these considerations motivated further study of oriented $\mathrm{Sb}_{2} \mathrm{Se}_{3}$ films and devices herein.

$\mathrm{Sb}_{2} \mathrm{Se}_{3}$ has a low melting point of $608{ }^{\circ} \mathrm{C}$ and a high saturated vapor pressure $\left(\sim 1200 \mathrm{~Pa} \text { at } 550{ }^{\circ} \mathrm{C}\right)^{20}$, 
enabling us to carry out film deposition using rapid thermal evaporation (RTE) in a tube furnace with high ramp rate (supplementary Fig. $\mathrm{S} 1$ ). $\mathrm{Sb}_{2} \mathrm{Se}_{3}$ powder was directly applied via evaporation under low vacuum pressure ( 8 mTorr), maintained by a mechanical pump. Once heated up, $\mathrm{Sb}_{2} \mathrm{Se}_{3}$ powder evaporated and condensed on the substrate because of temperature gradient, forming the $\mathrm{Sb}_{2} \mathrm{Se}_{3}$ film. The distance between the evaporating source and the substrate was kept at a low value of $0.8 \mathrm{~cm}$ to enable high material usage and a fast deposition rate. The deposition rate was as high as $1 \mu \mathrm{m} / \mathrm{min}$, much greater than regular thermal evaporation (typically $0.01-0.1 \mu \mathrm{m} / \mathrm{min}$ ) or sputtering (typically 0.01-0.05 $\mu \mathrm{m} / \mathrm{min}$ ) and comparable to confined space sublimation (CSS) ${ }^{21}$, a key technology that has enabled the high manufacturing throughput and commercial success of CdTe solar cells. The RTE process is distinct from CSS since, in the RTE performed herein, $\mathrm{Sb}_{2} \mathrm{Se}_{3}$ melts and evaporates from the liquid phase, in contrast with direct sublimation from the solid. Using this simple and fast technology, phase-pure $\mathrm{Sb}_{2} \mathrm{Se}_{3}$ film (Supplementary Fig. S2) and solar cells were readily fabricated with high reproducibility.

We first analyzed using transmission electron microscopy (TEM) specific regions of the $\mathrm{Sb}_{2} \mathrm{Se}_{3}$ films made in actual solar devices. The full device stack consisted of a FTO substrate, a thin $(\sim 60 \mathrm{~nm}) \mathrm{CdS}$ layer produced through chemical bath deposition, an approximately $390 \mathrm{~nm}$ thick $\mathrm{Sb}_{2} \mathrm{Se}_{3}$ absorber layer deposited by the RTE process, and top Au electrodes from thermal evaporation. For TEM characterization, the sample was prepared by cross-sectioning from the certified device using focused ion beam. The cross-sectional TEM image showed that the $\mathrm{Sb}_{2} \mathrm{Se}_{3}$ film was compact and composed of large $\mathrm{Sb}_{2} \mathrm{Se}_{3}$ grains whose size equals the film thickness (Fig. 2a). To check whether the grains were single-crystalline, we applied high-resolution transmission electron microscopy (HRTEM) to analyze three arbitrarily-selected points (I, II and III in Fig. 2a) with corresponding lattice fringes in Fig. 2b, 2c and $2 \mathrm{~d}$. The distances between lattice lines was measured to be $0.320 \mathrm{~nm}$ and $0.318 \mathrm{~nm}$, corresponding to the separation of $(21 \overline{1})$ and (211) planes in orthorhombic $\mathrm{Sb}_{2} \mathrm{Se}_{3}$. The crystal planes extend continuously from the top to the bottom of the active region of the device, confirming that this grain was single-crystalline. This trend was confirmed with further study of multiple grains (Supplementary Fig. S3), reinforcing the picture that the $\mathrm{Sb}_{2} \mathrm{Se}_{3}$ films are made up of single crystalline grains.

Orientational control of the $\mathrm{Sb}_{2} \mathrm{Se}_{3}$ film is predicted to be crucial to realize efficient carrier transport and benign GBs. We investigated therefore the possibility of correlation between $\mathrm{Sb}_{2} \mathrm{Se}_{3}$ film orientation and photovoltaic device performance. Different substrate temperatures during the RTE process were explored maintaining identical $\mathrm{Sb}_{2} \mathrm{Se}_{3}$ film thickness. Devices of Class A were deposited onto substrate kept at $300^{\circ} \mathrm{C}$, and devices of Class B were deposited onto $350^{\circ} \mathrm{C}$ substrate. Both devices had an area of $0.095 \mathrm{~cm}^{2}$ defined by the gold electrodes. Typical device performance (Fig. 3a) measured under $100 \mathrm{~mW} / \mathrm{cm}^{2}$ illumination (class 3A solar simulator) reveals short-circuit current density $\left(J_{S c}\right)$ for Device A of $27.2 \mathrm{~mA} / \mathrm{cm}^{2}$, fill factor $(\mathrm{FF})$ of $53 \%$ and series resistance $\left(\mathrm{R}_{\mathrm{S}}\right)$ of $40 \Omega$; while the corresponding values in Device B is $18.4 \mathrm{~mA} / \mathrm{cm}^{2}, 47 \%$ and $89 \Omega$. We first applied energy dispersive X-ray spectroscopy (EDX) (Supplementary Fig. S4 and Table S1-3) and capacitance-voltage (Supplementary Fig. S5) to analyze our devices and found out that both the $\mathrm{Sb}_{2} \mathrm{Se}_{3}$ films deposited at substrate temperature of $300^{\circ} \mathrm{C}$ and $350^{\circ} \mathrm{C}$ were slightly Se-rich (molar ratio Se: Sb is 1.51) and 
showed very close doping density. This indicated that film composition and consequent doping density likely did not account for the observed device efficiency difference. The large improvement from device Class B (3.2\% efficiency) to Class A (5.6\% efficiency) cannot be accounted for by stoichiometry/compositional differences, and we instead ascribe it to different film orientation. Indeed for device Class A, the diffraction intensity associated with the (120) peak is much weaker than for the (211) peak, while in device Class B the intensities of these two peaks are comparable with each other (Fig. 3b).

To quantitate orientation effects, we calculated the texture coefficient of the (120) peaks from 140 devices and plotted the corresponding device efficiency versus texture coefficient in Fig. 3c. The texture coefficient measures film orientation (Supplementary Fig. S6), with large texture coefficients for a diffraction peak indicating preferred orientation along this direction ${ }^{22}$. There exists a strong correlation between device performance and film orientation: device efficiency monotonically decreases when the texture coefficient of (120) orientation increases. Further analysis revealed that for all of these devices, the shunt resistance $\left(\mathrm{R}_{\mathrm{Sh}}\right)$ remains constant at approximately $1600 \Omega$, confirming similar junction quality, while the series resistance increased monotonically with an increased value of the (120) texture coefficient. We can explain this observation - that preferred orientation along the [120] direction resulted in significantly increased series resistance and decreased device efficiency - by noting that the [120] orientated grain consists of $\left(\mathrm{Sb}_{4} \mathrm{Se}_{6}\right)_{n}$ ribbons horizontally stacked in parallel with the substrate (Fig. 3d). In contrast, [221] orientated grain consists of tilted $\left(\mathrm{Sb}_{4} \mathrm{Se}_{6}\right)_{\mathrm{n}}$ ribbons stacked vertically on the substrate. It should be noted that the [211] orientated grain is also composed of tilted $\left(\mathrm{Sb}_{4} \mathrm{Se}_{6}\right)_{\mathrm{n}}$ ribbons, but to a different angle, thus we limit our transport discussion to the [221] orientated grain. Naturally, carrier transport in the [211] orientated grains should be much easier than in the [120] orientated grains because in the former ones carriers travel within the covalently bonded $\left(\mathrm{Sb}_{4} \mathrm{Se}_{6}\right)_{\mathrm{n}}$ ribbons, while in the latter ones they are required to hop between ribbons held together by van der Waals forces. Furthermore, the GBs of the [211] orientated grains are composed of low surface energy (hk0) planes such as (100), (010), (110), (120) planes, which are free of dangling bonds and should cause little recombination loss. In sum, we explain lower series resistance, better fill factor, and higher short-circuit current as resulting from improved transport and lowered recombination loss observed in devices having preferred [211] orientation of $\mathrm{Sb}_{2} \mathrm{Se}_{3}$ active layers.

For polycrystalline thin film solar cells, passivation of GBs to suppress strong carrier recombination is mandatory to achieve high efficiency devices. Typical examples are the carefully engineered $\mathrm{Cu}$ deficient GBs in CIGS solar cells ${ }^{23,24}$ and a high temperature $\mathrm{CdCl}_{2}$ treatment for CdTe solar cells ${ }^{24,25}$. We studied the properties of GBs in our $\mathrm{Sb}_{2} \mathrm{Se}_{3}$ solar cells using Kelvin probe force microscope (KPFM) and electron-beam-induced current (EBIC) measurements. Two-dimensional topography spatial maps and the corresponding surface potentials of $\mathrm{Sb}_{2} \mathrm{Se}_{3}$ thin films (Fig. 4a and 4b) reveal that there is no correlation between GBs (identifiable by notable changes in surface topography) with substantial potential variation in KFPM image. Overall, the average roughness of $\mathrm{Sb}_{2} \mathrm{Se}_{3}$ film is $23 \mathrm{~nm}$, while the average surface potential difference is a very low (much below kT) $9.1 \mathrm{mV}$. The surface potential fluctuations are extremely small compared to CIGS and CZTS films (generally $>100 \mathrm{mV}$ in a $2.5 \mu \mathrm{m} \mathrm{x}$ $2.5 \mu \mathrm{m}$ zone $\mathrm{e}^{9,25}$ ). In an illustrative line scan crossing the GBs (Fig. 4c), the surface potential difference between two grains is as low as $10 \mathrm{mV}$, indicating a lack of significant band bending and surface defects in the $\mathrm{Sb}_{2} \mathrm{Se}_{3}$ films. 
Further scanning electron microscopy (SEM) and EBIC analysis of $\mathrm{Sb}_{2} \mathrm{Se}_{3}$ solar cells are reported in Fig. 4d and 4e, respectively. The thickness of $\mathrm{Sb}_{2} \mathrm{Se}_{3}$ and CdS layer was $390 \mathrm{~nm}$ and $60 \mathrm{~nm}$ respectively, which is consistent to the results obtained from TEM characterization (Fig. 2a). Bright areas in an EBIC image indicate regions of high collection efficiency for minority carriers. Whereas in CdTe and CIGS, high contrast indicates non-uniform collection efficiency ${ }^{26}$, the case of $\mathrm{Sb}_{2} \mathrm{Se}_{3}$ solar cell exhibits no appreciable variation in EBIC signal across the absorber layer close to the CdS layer (Fig. 4e) and more importantly also across a $2 \mu \mathrm{m}$ long line scan (Fig. 4f). Since we showed previously that the same materials processing led to $\sim 300 \mathrm{~nm}$ grain sizes, these results indicate that the EBIC signal, hence collection efficiency, is uniform over multiple grains, excluding severe carrier recombination or efficient current colleting path presented at GBs. The EBIC findings echo the KFPM results above and clearly demonstrates that the GBs and grain interior are indistinguishable in our $\mathrm{Sb}_{2} \mathrm{Se}_{3}$ film, an expected consequence of the dangling-bond-free GBs.

We obtained certification of the solar cells reported herein (Fig. 5a and 5b, Newport Certificate Number 1017). In all devices reported herein, illumination is from the FTO side, and photogenerated carriers separate at the $\mathrm{p}-\mathrm{Sb}_{2} \mathrm{Se}_{3} / \mathrm{n}$-CdS interface. Electrons are injected into $\mathrm{CdS}$ and collected by FTO, and holes travel through the $\mathrm{Sb}_{2} \mathrm{Se}_{3}$ absorber and are collected by the back Au ohmic contact. The certified device $\left(0.095 \mathrm{~cm}^{2}\right)$ exhibited a $J S c$ of $25.1 \mathrm{~mA} / \mathrm{cm}^{2}$, a $V o c$ of $0.40 \mathrm{~V}$, and a fill factor of $55.7 \%$, corresponding to a power conversation efficiency of $5.6 \%$ (Fig. 5a). This agrees with in-house measurements, and in each case no hysteresis between forward and reverse scans was observed (Supplementary Fig. S7). We also built large-area $\left(1.08 \mathrm{~cm}^{2}\right)$ devices and obtained device efficiency of $5.4 \%$ measured with the aid of mechanical scribing and aperture (Supplementary Fig. S8-9). This device efficiency surpasses that of many other emerging inorganic solar cells based on Cd-free active absorbers, such as $\mathrm{SnS}^{27}$ and $\mathrm{FeS}_{2}{ }^{28}$. External quantum efficiency (EQE) measurement showed photocurrent contribution extended to $1070 \mathrm{~nm}$, consistent with the measured optical band gap. By dividing the EQE spectrum by the optical absorption, we obtained an internal quantum efficiency (IQE) close to $100 \%$ in the $550-600 \mathrm{~nm}$ solar spectrum. The high IQE is again consistent with low recombination loss assigned to the benign GBs studied in detail herein. The photovoltaic performance obtained across a study of 100 devices (Fig. 5c) demonstrates a tight distribution with an average efficiency of $5.24 \%$ and standard deviation of $0.34 \%$. Device efficiency (Fig. 5d) increased about 0.3 power points (from $5.32 \%$ to $5.66 \%$ ) when stored in air ambient for up to 45 days without encapsulation, in good agreement with our previous report of thermally evaporated $\mathrm{Sb}_{2} \mathrm{Se}_{3}$ solar cells ${ }^{19,29}$. Damp-heat testing $\left(85^{\circ} \mathrm{C}\right.$ and $85 \%$ humidity, no encapsulation) of the same device showed a slightly reduced device performance, from an initial $5.67 \%$ efficiency to $5.39 \%$ after $10 \mathrm{~h}$ testing and to $5.16 \%$ after $100 \mathrm{~h}$ testing. This amount of degradation is considerably lower than many next-generation solar cells such as organic-inorganic halide perovskite and polymer solar cells ${ }^{5}$.

The path to improved solar power conversion efficiency requires major progress on current (from 25 towards a maximum available $42 \mathrm{~mA} / \mathrm{cm}^{2}$ for the $1.1 \mathrm{eV}$ bandgap), voltage (from 0.4 to $0.75 \mathrm{~V}$ for this bandgap), and fill factor (from 50\% range into $80 \%$ range). The low EQE at short wavelengths (Fig. 5b) suggests that the CdS front layer should be replaced by a more desirable junction-forming material with 
larger bandgap and environmentally-friendly composition such as $\mathrm{TiO}_{2}$ or $\mathrm{ZnO}$. While ambipolar transport is efficiently achieved over $\sim 150 \mathrm{~nm}$ (viz. > 80\% EQE at wavelength $600 \mathrm{~nm}$ at which absorption length is $\sim 150 \mathrm{~nm}$ ), these materials and devices require in-depth study to determine whether the minority carrier diffusion length requires extending, or whether an electron-blocking back contact (back surface field) is the major requirement for improved current. The modest Voc and FF combined with the evidence for benign GBs suggests that electrical interfaces to $\mathrm{Sb}_{2} \mathrm{Se}_{3}$ - for efficient charge extraction, maximal quasi-Fermi-level splitting, and blocking of one carrier at each interface - deserve significant attention.

In sum, this work presented a non-toxic, stable, manufacturable method of solar cell fabrication that, in initial trials, led to a $5.6 \%$ certified device efficiency. The work reveals that materials with a 1D crystal structure, little explored in photovoltaics, offer promise as absorbers once the films are orientated suitably for transport. We showed that these materials can simultaneously sustain excellent transport along one axis and minimal recombination (due to benign grain boundaries) along the orthogonal axes. The family of $1 \mathrm{D}$ materials include other $\mathrm{V}_{2}-\mathrm{VI}_{3}$ compound such as $\mathrm{Bi}_{2} \mathrm{~S}_{3}$ and V-VI-VII compound such as SbSeI which all possess attractive material and optoelectronic properties. Analogously, 2D binary layered materials such as $\mathrm{MoSe}_{2}$ and $\mathrm{WSe}_{2}$, when similarly aligned to a substrate, are expected to exhibit sufficient transport and manifest the benefits of covalently-bonded planes and partially benign GBs.

\section{Methods}

Solar cell fabrication: All devices were deposited on glass coated $\mathrm{SnO}_{2}: \mathrm{F}$ supplied by Kaivo (Zhuhai, China) that had been cleaned using detergent, acetone, isopropanol and water rinse in sequence.

Chemical bath deposition $\left(\mathrm{CdSO}_{4}, 65^{\circ} \mathrm{C}, 16 \mathrm{~min}\right)$ was used to deposit the $\mathrm{CdS}$ layer of about $60 \mathrm{~nm}$ thickness. A $\mathrm{CdCl}_{2}$ post-treatment was applied to the $\mathrm{CdS}$ buffer layer: 1) $\mathrm{CdCl}_{2}$ (99\%, Aladdin)-anhydrous methanol saturated solution (about $20 \mathrm{mg} / \mathrm{ml}$ ) was spin-coated onto the CdS layer and then rinsed by methanol; 2) the treated CdS layer was baked on a hot plate at $400{ }^{\circ} \mathrm{C}$ for 5 min in air. $\mathrm{Sb}_{2} \mathrm{Se}_{3}$ layer was deposited by rapid thermal evaporation in a tube furnace (MTI, Hefei, China). The setup is schematically showed in Supplementary Fig. S1. $\mathrm{Sb}_{2} \mathrm{Se}_{3}$ powder was placed on the AlN plate inside the quartz boat, and FTO/CdS substrate was placed on top the quartz boat $(0.8 \mathrm{~cm}$ distance from the $\mathrm{Sb}_{2} \mathrm{Se}_{3}$ powder) with $\mathrm{CdS}$ side facing down. When pressure was reduced below 10 mtorr, film deposition was initiated. The deposition process was: preheat the source and substrate at $300{ }^{\circ} \mathrm{C}$ for 15 min, then ramp temperature to approximately $550{ }^{\circ} \mathrm{C}$ within $30 \mathrm{sec}$, maintain this temperature for 30 sec, and then turn off heating and allow the film to naturally cool down to $\sim 150^{\circ} \mathrm{C}$. Films were then taken out for Au deposition. Gold back-contacts $\left(0.095 \mathrm{~cm}^{2}\right)$ were thermally evaporated using the electron beam and resistance evaporation thin film deposition system (Beijing Technol Science Co. Ltd.) under a vacuum pressure $5 \times 10^{-3} \mathrm{~Pa}$.

Solar cell performance measurement: The devices were measured under simulated $100 \mathrm{~mW} / \mathrm{cm}^{2}$ AM1.5G irradiation which is generated through a 3A solar simulator with a Xe light source (450W, Oriel, Model 9119). Keithley 2400 was applied to measure the current-voltage characteristics in air. Device area $\left(0.095 \mathrm{~cm}^{2}\right)$ was defined by the size of the gold electrodes. For in-house measurement, no 
temperature control was applied. The scan rate was $0.1 \mathrm{~V} / \mathrm{s}$; no hysteresis was observed between forward (Jsc to Voc) and reverse (Voc to Jsc) scan (Supplementary Fig. S7). We also applied aperture (using a metal mask to define the incident light) and mechanical scribing to measure some of devices and obtained comparable device efficiencies (Supplementary Fig. S8-9). The device was further independently certified by Newport cooperation, American (Certificate Number 1017 issued on May 16, 2014, without aperture). The aging of the devices under $85{ }^{\circ} \mathrm{C}$ with $85 \%$ humidity was applied in a desktop constant temperature and humidity testing machine QA-HPZ-30 (Dongguan Cheng Yang Instruments Industry Co. Ltd.).

X-ray diffraction: XRD measurements were performed using a Philips diffractometer (X pert pro MRD with a step of $0.017^{0}$ and step time of 10.16 s. The lines used are $\mathrm{Cu} \mathrm{K}_{\alpha 1}$ and $\mathrm{Cu} \mathrm{K}_{\alpha 2}$ with wavelengths of $1.54060 \mathrm{~nm}$ and $1.54443 \mathrm{~nm}$, respectively.

TEM and SAED characterization: Samples for TEM and SAED analysis were prepared by ablating the certified device using a FEI Quanta 3D FEG-FIB. A thin Pt layer was first deposited on top of the device for protection. TEM images and SAED measurements were taken using a FEI Titan G2 60-300 Probe Cs Corrector STEM operating at $300 \mathrm{kV}$.

Kelvin probe force microscopy: KFPM was performed on a grounded $\mathrm{Sb}_{2} \mathrm{Se}_{3}$ sample using a NT-MDT dimension 3100 system. Topography and surface potential were simultaneously measured using a Pt-coated silicon probe (NT-MDT, HQ: NSG11/Pt, $f_{0} \sim 150 \mathrm{kHz}, k \sim 5.5 \mathrm{~N} / \mathrm{m}$, sampling rate 0.25 $\mathrm{Hz}$ ). Surface potential was calculated from the electrostatic force between the probe tip and the surface of the sample with the use of a lock-in amplifier. The scanning area was $2.5 \mu \mathrm{m} \times 2.5 \mu \mathrm{m}$ of $256 \times 256$ pixels. The minimal distance between two scanned points was about $5 \mathrm{~nm}$, and the potential measurement limit and resolution is $10 \mathrm{mV}$ and $1 \mathrm{mV}$, respectively.

Electron-beam-induced current measurement: The device was cleaved by hand after scribing on the back and loaded into scanning electron microscope vacuum chamber for EBIC measurement. SEM images were taken on a Quanta 400 FEG, FEI microscope equipped with a SmartEBIC (Gatan, Inc.). The gold back contact was contacted to the preamplifier by a small micromanipulator to enable current measurement. The e-beam voltage is $20 \mathrm{kV}$ and the working distance is about $10.5 \mathrm{~mm}$. Images were processed using the Digital micrograph software.

Simulation methods: The structural relaxation and electronic structure of the $\mathrm{Sb}_{2} \mathrm{Se}_{3}$ are calculated within the density functional formalism as implemented in the VASP $\operatorname{code}^{30}$. For the exchange-correlation potential, we used the non-local vdW-DF method proposed by Dion et al. to describe the van der Waals interaction. The interaction between the core electrons and the valence electrons is included by the frozen-core projector augmented-wave (PAW) pseudopotentials. An energy cut-off of $400 \mathrm{eV}$ was applied in all cases. For Brillouin-zone integration, we used the $4 \times 4 \times 12$ Monkhorst-Pack k-point mesh for the 20 -atom primitive cell, and $2 \times 6 \times 1(2 \times 2 \times 1)$ meshes for the $(100)$ [(010)] surface slab models. The lattice vectors of the primitive cell and the atomic positions were fully relaxed by minimizing the quantum mechanical stresses and forces.

\section{Reference:}

1. Wang, W. et al. Device Characteristics of CZTSSe Thin-Film Solar Cells with $12.6 \%$ Efficiency. Adv. Energy Mater. 4, 1301465 (2014).

2. Jeon, N. J. et al. Solvent engineering for high-performance inorganic-organic hybrid perovskite solar cells. Nature Mater. 13, 897-903 (2014).

3. Chen, S., Walsh, A., Gong, X. G. \& Wei, S. H. Classification of lattice defects in the kesterite 
$\mathrm{Cu}_{2} \mathrm{ZnSnS}_{4}$ and $\mathrm{Cu}_{2} \mathrm{ZnSnSe}_{4}$ earth-abundant solar cell absorbers. Adv. Mater. 25, 1522-1539 (2013).

4. Barkhouse, D. A. R., Gunawan, O., Gokmen, T., Todorov, T. K. \& Mitzi, D. B. Device characteristics of a $10.1 \%$ hydrazine-processed $\mathrm{Cu}_{2} \mathrm{ZnSn}(\mathrm{Se}, \mathrm{S})_{4}$ solar cell. Prog. Photovolt. 20, 6-11 (2012).

5. Gratzel, M. The light and shade of perovskite solar cells. Nature Mater. 13, 838-842 (2014).

6. Kranz, L. et al. Doping of polycrystalline CdTe for high-efficiency solar cells on flexible metal foil. Nature Commun. 4, 2306 (2013).

7. Panthani, M. G. et al. Synthesis of $\mathrm{CuInS}_{2}, \mathrm{CuInSe} 2$, and $\mathrm{Cu}\left(\operatorname{In}_{\mathrm{x}} \mathrm{Ga}_{1-\mathrm{x}}\right) \mathrm{Se}_{2}$ (CIGS) Nanocrystal “Inks” for Printable Photovoltaics. J. Am. Chem. Soc. 130, 16770-16777 (2008).

8. Chirilă, A. et al. Potassium-induced surface modification of $\mathrm{Cu}(\mathrm{In}, \mathrm{Ga}) \mathrm{Se}_{2}$ thin films for high-efficiency solar cells. Nature Mater. 12, 1107-1111 (2013).

9. Baier, R., Leendertz, C., Abou-Ras, D., Lux-Steiner, M. C. \& Sadewasser, S. Properties of electronic potential barriers at grain boundaries in $\mathrm{Cu}(\mathrm{In}, \mathrm{Ga}) \mathrm{Se}_{2}$ thin films. Sol. Energ. Mat. Sol. $C$. 130, 124-131 (2014).

10. Choi, Y. C., Lee, D. U., Noh, J. H., Kim, E. K. \& Seok, S. I. Highly Improved $\mathrm{Sb}_{2} \mathrm{~S}_{3}$ Sensitized-Inorganic-Organic Heterojunction Solar Cells and Quantification of Traps by Deep-Level Transient Spectroscopy. Adv. Funct. Mater. 24, 3587-3592 (2014).

11. Zhang, S., Wei, S.-H., Zunger, A. \& Katayama-Yoshida, H. Defect physics of the CuInSe $\mathrm{C}_{2}$ chalcopyrite semiconductor. Phys. Rev. B 57, 9642 (1998).

12. Leite, M. S. et al. Nanoscale Imaging of Photocurrent and Efficiency in CdTe Solar Cells. ACS nano 8, 11883-11890 (2014).

13. Yin, W. J., Shi, T. \& Yan, Y. Unique properties of halide perovskites as possible origins of the superior solar cell performance. Adv. Mater. 26, 4653-4658 (2014).

14. Tang, J. et al. Colloidal-quantum-dot photovoltaics using atomic-ligand passivation. Nature Mater. 10, 765-771 (2011).

15. Schmidt, J. et al. Surface passivation of high-efficiency silicon solar cells by atomic-layer-deposited $\mathrm{Al}_{2} \mathrm{O}_{3}$. Prog. Photovolt. 16, 461-466 (2008).

16. Choi, Y. C. et al. $\mathrm{Sb}_{2} \mathrm{Se}_{3}$ sensitized inorganic-organic heterojunction solar cells fabricated using a single-source precursor. Angew. Chem. Int. Edit. 53, 1329-1333 (2014).

17. Zhou, Y. et al. Solution-Processed Antimony Selenide Heterojunction Solar Cells. Adv. Energy Mater. 4 (2014).

18. Patrick, C. E. \& Giustino, F. Structural and Electronic Properties of Semiconductor-Sensitized Solar-Cell Interfaces. Adv. Funct. Mater. 21, 4663-4667 (2011).

19. Luo, M. et al. Thermal evaporation and characterization of superstrate $\mathrm{CdS} / \mathrm{Sb}_{2} \mathrm{Se}_{3}$ solar cells. Appl. Phys. Lett. 104, 173904 (2014).

20. Liu, X. et al. Thermal Evaporation and Characterization of $\mathrm{Sb}_{2} \mathrm{Se}_{3}$ Thin Film for Substrate $\mathrm{Sb}_{2} \mathrm{Se}_{3} / \mathrm{CdS}$ Solar Cells. ACS Appl. Mater. Inter. 6, 10687-10695 (2014).

21. Major, J., Treharne, R., Phillips, L. \& Durose, K. A low-cost non-toxic post-growth activation step for CdTe solar cells. Nature 511, 334-337 (2014).

22. Mashtalir, O. et al. Intercalation and delamination of layered carbides and carbonitrides. Nature Commun. 4, 1716 (2013).

23. Hetzer, M. et al. Direct observation of copper depletion and potential changes at copper indium gallium diselenide grain boundaries. Appl. Phys. Lett. 86, 162105 (2005). 
24. Jiang, C.-S. et al. Local built-in potential on grain boundary of $\mathrm{Cu}$ (In, Ga) $\mathrm{Se} 2$ thin films. Appl. Phys. Lett. 84, 3477-3479 (2004).

25. Li, J. B., Chawla, V. \& Clemens, B. M. Investigating the role of grain boundaries in CZTS and CZTSSe thin film solar cells with scanning probe microscopy. Adv. Mater. 24, 720-723 (2012).

26. Li, C. et al. Grain-Boundary-Enhanced Carrier Collection in CdTe Solar Cells. Phys. Rev. Lett. 112, 156103 (2014).

27. Sinsermsuksakul, P. et al. Overcoming Efficiency Limitations of SnS - Based Solar Cells. Adv. Energy Mater. 4 (2014).

28. Limpinsel, M. et al. An inversion layer at the surface of n-type iron pyrite. Energy. Environ. Sci. 7, 1974 (2014).

29. Leng, M. et al. Selenization of $\mathrm{Sb}_{2} \mathrm{Se}_{3}$ absorber layer: An efficient step to improve device performance of $\mathrm{CdS} / \mathrm{Sb}_{2} \mathrm{Se}_{3}$ solar cells. Appl. Phys. Lett. 105, 083905 (2014).

30. Kresse, G. \& Furthmüller, J. Efficient iterative schemes for ab initio total-energy calculations using a plane-wave basis set. Phys. Rev. B 54, 11169 (1996).

\section{Acknowledgements}

This work is supported by the director fund of Wuhan National Laboratory for Optoelectronics (WNLO), the National 1000 Young Talents project, National Natural Science Foundation of China (NSFC 61274055, 91233121, 91433105, 21403078) and the 973 program of China (2011CBA00703). The authors would like to thank the Analytical and Testing Center of HUST, the Center for Nanoscale Characterization and Devices of WNLO and the Suzhou Institute of Nano-Tech and Nano-Bionics (SINANO) for the characterization support. Prof. Yanfa Yan at University of Toledo and Prof. Haizheng Zhong at Beijing Institute of Technology are acknowledged for helpful discussions.

\section{Author Contribution}

Y. Z and J. T conceived the idea, designed the experiments and analyzed the data. Y. Z and L. W carried out most characterizations and device optimizations. S. Y. C performed the theoretical simulation and analyzed the results. S. K and X. L initialized the RTE process. J. C, D. X, M. L, Y. Z. C participated in device optimization and data analysis. Y. B. C. helped manuscript preparation. E. H. S. and J. T wrote the paper; all authors commented on the manuscript.

\section{Additional Information}

Supplementary information is available in the online version of the paper. Reprints and permissions information is available online at www.nature.com/reprints. Correspondence and requests for materials should be addressed to J. T. (jtang@ mail.hust.edu.cn).

\section{Competing financial interests}

The authors declare no competing financial interests.

\section{Legends}

Figure 1 | Crystal structure, density of states, and recombination loss at GBs in CdTe and $\mathrm{Sb}_{2} \mathrm{Se}_{3}$ solar cells. (a) CdTe possesses 3D crystal structure (zinc blende) and has dangling bonds (shown as red rods) at GBs, which 
act as defects causing recombination loss of photogenerated carriers. (b) $\mathrm{Sb}_{2} \mathrm{Se}_{3}$ (orthorhombic) is composed of $\left(\mathrm{Sb}_{4} \mathrm{Se}_{6}\right)_{\mathrm{n}}$ ribbons stacking in parallel in the [001] direction. All atoms at the edge of these ribbons are saturated (highlighted as red spheres) and introduce no recombination loss at GBs once they are vertically orientated onto the substrates. Note we omitted in the image $\left(\mathrm{Sb}_{4} \mathrm{Se}_{6}\right)_{n}$ ribbons that are present underneath yet in a staggered manner for clarity. (c) Atomic configuration of the (010) and (100) surfaces (marked by the dashed black grids) in $\mathrm{Sb}_{2} \mathrm{Se}_{3}$ crystal. (d) The calculated density of states of bulk $\mathrm{Sb}_{2} \mathrm{Se}_{3}$ and four surfaces that are parallel to [001] direction. The forbidden gap is the empty zone near $0 \mathrm{eV}$.

Figure 2 | Device's structure and TEM analysis of $\mathbf{S b}_{2} \mathrm{Se}_{3}$ films and devices. (a) Cross-sectional TEM image of the certified device. The nature of each layer and the corresponding preparation procedures are included in the right-hand legend. The inset also shows the picture of a finished device; the 9 yellow squares are the gold electrodes. HRTEM scans were performed at points I, II and III, and the corresponding lattice fringes are shown in panel (b), (c) and (d).

Figure 3 | Device performance and its correlation with crystalline orientation. Solar cells with different photovoltaic performance (a) and the corresponding XRD patterns (b). The standard diffraction pattern for $\mathrm{Sb}_{2} \mathrm{Se}_{3}$ (JCPDS 15-0861) is included for reference. The asterisk marks the peaks indexed to the FTO substrate. The peak at $13^{0}$ is associated by indexing to trace elemental Se excess. (c) Statistics of the texture coefficient of (120) peaks with the device efficiency, series resistance (Rs, left axis in the inset) and shunt resistance (Rsh, right axis in the inset) of corresponding devices. 140 devices are included in obtaining the statistics presented. (d) Atomic structure of [120] and [221] orientated grains in $\mathrm{Sb}_{2} \mathrm{Se}_{3}$. Substrate means the CdS buffer layer where $\mathrm{Sb}_{2} \mathrm{Se}_{3}$ ribbons grow. Upon photoexcitation, charge carriers must hop between ribbons in the [120] orientated grain (dashed red lines) but are able to move along the [221] orientated grain smoothly (solid red lines).

Figure 4 | Surface potential at $\mathrm{Sb}_{2} \mathrm{Se}_{3}$ GBs and EBIC images from a crystallographically well oriented device (deposited onto $300^{\circ} \mathrm{C}$ substrates). (a) Atomic Force Microscopy (AFM) topography and (b) scanning KPFM image of the device. Scanning area is $2.5 \mu \mathrm{m} \times 2.5 \mu \mathrm{m}$. (c) Contact potential difference and height along the solid black lines drawn in panel a and b. The valleys of the height curve correspond to the GBs. (d) Cross-sectional SEM and (e) EBIC images for the cleaved $\mathrm{Sb}_{2} \mathrm{Se}_{3}$ devices. For the colored legend, a is $\mathrm{Au}, \mathrm{b}$ is $\mathrm{Sb}_{2} \mathrm{Se}_{3}, \mathrm{c}$ is $\mathrm{CdS}$ and d is FTO. White parts indicate zones where electron beam induced carriers were efficiently collected, while grey areas mean the $\mathrm{Sb}_{2} \mathrm{Se}_{3}$ zones contributing less photocurrent. (f) The intensity profiling along the dashed blue line marked in panel e. The scattering in signal intensity is negligible in the scanned $1.5 \mu \mathrm{m}$ length, suggesting uniform photocurrent collecting efficiency even cross GBs.

Figure 5| Device performance (certified) and stability. (a) Current-voltage (I-V) and (b) EQE spectra of the $\mathrm{FTO} / \mathrm{CdS} / \mathrm{Sb}_{2} \mathrm{Se}_{3} / \mathrm{Au}$ solar cell independently certified by Newport cooperation (Calibration certificate \#1017). (c) Histogram of devices efficiencies obtained from over 100 individually fabricated devices: Voc $=0.385 \pm 0.012 \mathrm{~V}$, $\mathrm{Jsc}=25.47 \pm 1.75 \mathrm{~mA} / \mathrm{cm}^{2}, \mathrm{FF}=53.37 \pm 3.72 \%$ and the efficiencies were $5.24 \pm 0.34 \%$. (d) The stability of a typical device without package stored in regular laboratory conditions (ambient air, no shading) and then subjected to the damp-heat measurement $\left(85^{\circ} \mathrm{C}, 85 \%\right.$ humidity, in dark). 